\title{
UMA NOVA ABORDAGEM HEURÍSTICA PARA A RESOLUÇÃO DO PROBLEMA DO ROTEAMENTO DE VEÍCULOS CAPACITADOS
}

\section{A NEW HEURISTIC APPROACH TO SOLVE THE CAPACITATED VEHICLE ROUTING PROBLEM}

\author{
Thiago André Guimarães ${ }^{1}$; Cassius Tadeu Scarpin ${ }^{2}$; Maria Teresinha Arns Steiner ${ }^{3}$ \\ ${ }^{1}$ Universidade Federal do Paraná - UFPR - Curitiba - Brasil \\ thiandgui@gmail.com \\ ${ }^{2}$ Universidade Federal do Paraná - UFPR - Curitiba - Brasil \\ cassiusts@gmail.com \\ ${ }^{3}$ Universidade Federal do Paraná - UFPR - Curitiba - Brasil \\ tere@ufpr.br
}

\begin{abstract}
Resumo
O Problema do Roteamento de Veículos Capacitados (PRVC) é uma das mais conhecidas abordagens em pesquisa operacional para resolução de problemas em logística de distribuição. Por sua complexidade combinatória PRVC enseja o emprego de técnicas heurísticas para sua resolução. Neste sentido, este artigo apresenta uma nova abordagem heurística para a resolução do problema. O método emprega uma estratégia em dois estágios, que consiste primeiramente em agrupar os clientes de acordo com a demanda e, posteriormente, construir as rotas para os grupos formados. Para o primeiro estágio, foi desenvolvida uma heurística envolvendo os algoritmos clássicos de agrupamento e designação propostos por Teitz e Bart (1968) e Gillet Johnson (1976), respectivamente. No segundo estágio, as rotas iniciais são geradas pela heurística de inserção mais econômica e refinadas pelas heurísticas de melhoria 2-opt e 3-opt. A abordagem proposta foi testada para algumas instâncias clássicas da literatura e comparada com o desempenho de alguns procedimentos exatos e heurísticos, produzindo resultados interessantes, tanto em termos de eficácia quanto de eficiência.
\end{abstract}

Palavras-chave: roteamento de veículos capacitados; procedimentos heurísticos; agrupamento de pontos de demanda.

\section{Introdução}

O Problema do Roteamento de Veículos Capacitados (PRVC) vem sendo uma das mais importantes abordagens para a otimização de distribuição em redes, desde que foi proposto inicialmente por Dantzig e Ramser (1959). Tal estudo foi decorrente da análise de um problema de roteamento para distribuição de gasolina transportada por caminhões capacitados.

O PRVC busca determinar um conjunto de rotas para uma frota homogênea de veículos, partindo de um depósito central com destino a um conjunto de clientes que demandam determinado produto. Cada cliente deve ser atendido por apenas um veículo (embora haja variações no problema 
original que permite entregas fracionadas) e, além disso, a demanda transportada não deve exceder sua capacidade de carregamento. O objetivo do PRVC é minimizar a distância total percorrida pela frota de veículos.

Existem diversas abordagens para a resolução do PRVC. Em geral, essas abordagens podem ser agrupadas em duas classes distintas: métodos exatos e métodos heurísticos.

Referente à primeira classe, alguns dos trabalhos são baseados em algoritmos branch-andcut ou relaxação lagrangeana/geração de colunas. Um dos marcos na abordagem exata para o PRVC foi apresentado em Christofides et al. (1981), que trabalharam com limitantes lagrangeanos para a geração de subrotas. O algoritmo branch-and-bound foi capaz de resolver instâncias com até 25 clientes. Já, Fukasawa et al. (2006) desenvolveram um algoritmo branch-and-cut-and-price sob instâncias com mais de 135 clientes, obtendo para todas elas resultados ótimos.

Pesquisas recentes intensificam o emprego de planos de corte, como se verifica em Achuthan et al. (2003), que propuseram novos planos de corte baseados em um algoritmo branchand-cut e testaram sob instâncias que contêm uma faixa de 15 até 100 clientes, além de 24 problemas clássicos da literatura. O trabalho produziu benefícios em termos de esforço computacional.

Já em relação à segunda classe (métodos heurísticos), Campos e Mota (2000) apresentaram duas heurísticas: uma baseada em scratch, que gera uma solução inicial sem qualquer informação obtida a priori, e outra que emprega informações advindas de relaxações lineares fortes a partir do problema original. Os autores utilizaram técnicas de busca tabu para refinar as soluções iniciais. As heurísticas foram testadas sob instâncias da literatura com a quantidade de clientes variando de 22 a135 clientes, obtendo soluções ótimas para a maioria delas.

Berger e Bakaoui (2003) apresentaram um algoritmo genético híbrido, trabalhando com a evolução de duas populações ao mesmo tempo, utilizando operadores que combinam variações de técnicas de roteamento e técnicas de busca. Por sua vez, Chen et al. (2006) propuseram uma abordagem híbrida baseada em nuvem de partículas e testaram sob instâncias clássicas com uma faixa de clientes variando de 33 a 155. Os resultados obtidos se aproximaram dos valores ótimos.

Também empregando técnicas metaheurísticas, Zhishuo e Yueting (2005) desenvolveram um novo método baseado em colônia de formigas. O trabalho apresenta algumas inovações em relação às abordagens tradicionais da metaheurística para a resolução do PRVC, dentre elas o procedimento para a construção de subrotas, que são iniciadas randomicamente a partir dos vértices mais distantes ao invés de iniciarem nos depósitos. Na etapa de refinamento da solução atual, um algoritmo de varredura é aplicado sobre as subrotas, trocando pares de vértices entre elas. $\mathrm{O}$ trabalho apresentou resultados apenas $0,28 \%$ inferiores aos valores ótimos para as instâncias testadas, o que representa um desempenho superior entre outras abordagens baseadas em colônia de 
formigas para o PRVC.

Comumente é reportada na literatura uma estratégia em duas fases para a resolução do PRVC. Esta estratégia primeiramente agrupa os clientes conforme a localização e quantidade demandada para posteriormente construir rotas para os grupos formados (cluster first and then route). Garantido que a demanda dos clientes pertencentes a um grupo não exceda a capacidade do veículo, o PRVC recai na resolução do Problema do Caixeiro Viajante (PCV), ensejando um roteiro para cada grupo, que passe por todos os pontos, e apresente a menor distância possível. Fisher e Jaikumar (1981) e Gillett e Johnson (1976) endereçam heurísticas do tipo "cluster first and then route" para a resolução do PRVC. Variações do problema clássico tratadas com essa mesma abordagem encontram-se em Sariklis and Powell (2000), que trabalham para a resolução do OVRP (Open Vehicle Routing Problem). O OVRP se diferencia do PRVC pela não necessidade de retorno do veículo ao depósito após visitar o último cliente da rota.

Neste presente trabalho é proposta uma nova heurística híbrida para a resolução do PRVC. A abordagem emprega a supracitada estratégia em dois estágios: um primeiro estágio agrupa os clientes de acordo com suas localizações e respectivas demandas, considerando a capacidade do veículo, empregando a heurística clássica de Teitz e Bart (1968) em conjunto com o algoritmo de Gillet Johnson (1976). Neste estágio, uma nova heurística, denominada $k$-fit é apresentada, “ajustando" a heurística de Teitz e Bart a priori e o algoritmo de Gillet Johnson a posteriori. Já o segundo estágio roteiriza os agrupamentos, onde as subrotas iniciais são geradas pela heurística construtiva gulosa de inserção mais econômica e, posteriormente, são refinadas pelas heurísticas de melhoria 2-opt e 3-opt, respectivamente.

A abordagem proposta foi avaliada em instâncias clássicas da literatura e, além disso, comparada com outros procedimentos heurísticos e exatos propostos por outros autores. $\mathrm{O}$ artigo está dividido em cinco seções: a seção 2 descreve formalmente o problema, enquanto que a seção 3 apresenta a heurística proposta para a resolução do PRVC. A seção 4 apresenta os resultados testados sob instâncias da literatura e, por fim, a seção 5 tece as conclusões do artigo.

\section{Descrição do problema}

Seja $G(V, E)$ um grafo não direcionado contendo o conjunto de vértices $V=\{0,1, \ldots, n\}$, onde o vértice " 0 " representa o depósito, enquanto que todos os outros vértices representam os clientes, sendo que cada cliente $i$ possui uma demanda associada $d_{i}$. Cada $\operatorname{arco} e \in E$ possui um comprimento não negativo $\ell(e)$. Dado $G$ e dois números positivos e inteiros $(K$ e $Q)$, o PRVC consiste em encontrar um conjunto de rotas para os $K$ veículos, satisfazendo as seguintes restrições:

(i) Cada rota inicia e termina no depósito; 
(ii) Cada cliente é visitado por um único veículo;

(iii) A demanda total de todos os clientes de uma subrota não excede a capacidade $Q$ do veículo.

O objetivo do PRVC é minimizar o somatório das distâncias de todas as subrotas. O PRVC é fortemente $N P$-hard, dado que é uma generalização do PCV demandando, portanto, elevado esforço computacional para obtenção da solução ótima através de busca exaustiva. Baseado em Lin et al. (2009), o PRVC pode ser modelado como um problema de Programação Inteira Misto como segue:

$$
\begin{array}{ll}
\text { Minimizar } \quad & \sum_{i=0}^{N} \sum_{j=0}^{N} \sum_{k=1}^{K} C_{i j} X_{i j k} \\
\text { Sujeito à: } & \sum_{i=0}^{N} \sum_{j=0}^{N} X_{i j k} d_{i} \leq Q^{k} \quad 1 \leq k \leq K, \\
& \sum_{j=1}^{N} X_{i j k}=\sum_{j=1}^{N} X_{j i k} \leq 1, \text { para } i=0 \text { e } k \in\{1, \ldots, k\}, \\
& \sum_{k=1}^{K} \sum_{j=1}^{N} X_{i j k} \leq K, \text { para } i=0,
\end{array}
$$

Onde $C_{i j}$ é o custo para se ir do cliente $i$ para o cliente $j ; K$ é a quantidade de veículos disponível; $N$ é o número de clientes; $Q^{k}$ é a capacidade de carregamento do veículo $k ; d_{i}$ é a demanda do cliente $i$. As variáveis $X_{i j}^{k} \in 0$ ou $1(i \neq j ; i, j \in 0,1, \ldots, N)$.

A equação (1) é a função objetivo do problema que, conforme já comentado, minimiza o somatório das distâncias de todas as subrotas. As restrições (2) referem-se à capacidade de carregamento do veículo e atendimento das demandas de cada cliente, onde $X_{i j k}=1$, se o veículo $k$ viaja do cliente $i$ para o cliente $j$ diretamente e $X_{i j k}=0$, caso contrário. Já as restrições (3) garantem que toda rota tem início e fim no depósito, enquanto que a restrição (4) especifica que há no máximo $K$ rotas partindo do depósito.

Problemas de grande porte ensejam um elevado esforço computacional para sua resolução, dado que o tempo de processamento aumenta exponencialmente com o incremento do número de clientes (nós). Devido a isto, métodos heurísticos vêm sendo propostos para a resolução do PRVC. Neste sentido, a próxima seção discute a heurística aqui proposta.

\section{Heurística proposta}


A construção da heurística proposta neste presente artigo envolve: o problema de agrupamento; o problema de construção e melhorias de rotas. Assim, as próximas seções discutem esses problemas de forma pormenorizada para que, na seqüência, a heurística seja apresentada.

\subsection{O problema de agrupamento (clusterização)}

O problema das $p$-medianas é introduzido com o objetivo de determinar no grafo formado pelos clientes, $p$-pontos principais, que representem sementes ou depósitos fictícios. A cada uma destas medianas, os demais pontos de demanda serão designados, de modo a formar $p$-conjuntos iniciais ( $p$-grupos) de pontos, cada um com "capacidade" igual à capacidade de carregamento dos veículos, que é o mesmo para todos eles (frota homogênea). A estratégia de resolução reside na teoria dos grafos que tem por objetivo localizar facilidades (instalações) ao longo de uma rede viária. Estas facilidades ( $p$-medianas) devem ser escolhidas de forma a minimizar a soma da distância de cada um dos pontos à facilidade mais próxima, ponderada por um fator de demanda. Uma descrição mais formalizada para o problema é apresentada a seguir.

Para um grafo $G(V, E)$, define-se número de out-transmissão e in-transmissão, para cada ponto $v_{i} \in V$, como sendo, respectivamente:

$$
\begin{aligned}
& \sigma_{0}\left(v_{i}\right)=\sum_{v_{j} \in V} \theta_{j} \cdot w\left(v_{i}, v_{j}\right) \\
& \sigma_{t}\left(v_{i}\right)=\sum_{v_{j} \in V} \theta_{j} \cdot w\left(v_{j}, v_{i}\right)
\end{aligned}
$$

Onde, o vetor $w\left(v_{i}, v_{j}\right)$ é a distância entre o vértice $v_{i}$ e $v_{j}$, e $\theta_{j}$ é o peso associado ao ponto $v_{j}$. De acordo com Christofides (1975), são chamadas de out-mediana e in-mediana de um grafo, respectivamente, os pontos $\bar{v}_{i} e \bar{v}_{j}$ que satisfazem as seguintes condições:

$$
\begin{aligned}
& \sigma_{0}\left(\bar{v}_{0}\right)=\min _{v_{i} \in V}\left[\sigma_{0}\left(v_{i}\right)\right] \\
& \sigma_{t}\left(\bar{v}_{t}\right)=\min _{v_{i} \in V}\left[\sigma_{t}\left(v_{i}\right)\right]
\end{aligned}
$$

Para generalizar estes conceitos de out-transmissão e in-transmissão para $p$-medianas, considera-se $V_{p}$ um subconjunto do conjunto de pontos $V$ do grafo $G(V, E)$, que possui $p$ elementos, isto é, a cardinalidade de $V_{p}$ é $p$ (ou, simplesmente, $\left|V_{p}\right|=p$ ). Define-se ainda,

$$
w\left(V_{p}, v_{j}\right)=\min _{v_{i} \in V_{p}}\left[w\left(v_{i}, v_{j}\right)\right] \text { e } w\left(v_{j}, V_{p}\right)=\min _{v_{i} \in V_{p}}\left[w\left(v_{j}, v_{i}\right)\right]
$$


onde $w\left(V_{p}, v_{j}\right)$ representa a distância do subconjunto de pontos $V_{p}$ até o ponto $v_{j}$ e $w\left(v_{j}, V_{p}\right)$ indica a distância do vértice $v_{j}$ até o subconjunto $V_{p}$. Analogamente ao procedimento para um só vértice, definem-se os números de out-transmissão e in-transmissão, respectivamente, para o conjunto $V_{p}$, da seguinte forma:

$$
\begin{aligned}
& \sigma_{0}\left(V_{p}\right)=\sum_{v_{j} \in V} \theta_{j} \cdot w\left(V_{p}, v_{j}\right) \\
& \sigma_{t}\left(V_{p}\right)=\sum_{v_{j} \in V} \theta_{j} \cdot w\left(v_{j}, V_{p}\right)
\end{aligned}
$$

Finalmente, denomina-se de $p$-out-mediana e p-in-mediana os conjuntos $\bar{V}_{p o} e \bar{V}_{p t}$, respectivamente, para os quais:

$$
\begin{aligned}
& \sigma_{0}\left(\bar{V}_{p o}\right)=\min _{V_{p} \subset V}\left[\sigma_{0}\left(V_{p}\right)\right] \\
& \sigma_{t}\left(\bar{V}_{p t}\right)=\min _{V_{p} \subset V}\left[\sigma_{t}\left(V_{p}\right)\right]
\end{aligned}
$$

Neste trabalho, considera-se um grafo não orientado, tornando indiferente o conceito de $p$ in-mediana ou $p$-out-mediana, que doravante será denominado apenas de $p$-mediana, simplificando consideravelmente a notação utilizada.

O problema das $p$-medianas pode ser resolvido de forma exata (HAKIMI, 1965), usando enumeração exaustiva ou programação inteira. Todavia, para problemas de grande porte (grande quantidade de pontos), torna-se necessário recorrer a métodos aproximados (heurísticos) tendo em vista o esforço computacional requerido pelos métodos exatos ser muito elevado. Ainda que exista na literatura, uma vasta gama de abordagens heurísticas para a resolução do problema das $p$ medianas com uma grande quantidade de pontos, destaca-se o algoritmo proposto por Teitz e Bart (1968) pela eficiência, estabilidade e poder de generalização.

No supracitado algoritmo, inicialmente escolhe-se um conjunto $S$ formado por $p$ pontos, considerado como uma aproximação do conjunto $\bar{v}$ das medianas. Verifica-se se algum ponto $v_{i} \in(V-S)$ pode substituir, de acordo com o algoritmo apresentado a seguir, algum ponto $v_{j} \in V$, produzindo um novo conjunto $S^{\prime}$ tal que: $S^{\prime}=S \cup\left\{v_{i}\right\}-\left\{v_{j}\right\}$ e $\sigma\left(S^{\prime}\right)<\sigma(S)$. Se isto for possível, substituímos $v_{j}$ por $v_{i}$ e $S$ ' é considerado uma nova aproximação para o conjunto $S$, onde nenhuma substituição de pontos produza um número de transmissão menor. O algoritmo é detalhadamente descrito a seguir.

Passo 1. Construa um conjunto inicial $S, \operatorname{com} p$ elementos de $V$; 
Passo 2. Rotule todos os pontos $v_{i} \notin S$ como "não-analisados";

Passo 3. Enquanto existirem pontos "não-analisados” no conjunto $(V-S)$, faça:

a) Selecione um vértice "não-analisado" $v_{i} \in(V-S)$, e calcule a redução $\Delta_{i j}$ do número de transmissão, $\forall v_{j} \in S: \Delta_{i j}=\sigma(S)-\sigma\left(S \cup\left\{v_{i}\right\}-\left\{v_{j}\right\}\right)$;

Faça $\Delta_{i j o}=\max _{v_{j} \in S}\left\{\Delta_{i j}\right\}$;

b) Se $\Delta_{i j o}>0$ faça $S \leftarrow S \cup\left\{v_{i}\right\}-\left\{v_{j}\right\}$ rotulando $v_{j o}$ como "analisado";

c) Se $\Delta_{i j o} \leq 0$ rotule $v_{i}$ como "analisado".

Passo 4. Se durante a execução do passo 3 ocorrer modificações no conjunto $S$, volte para o passo 2. Caso contrário PARE. O conjunto $S$ será uma aproximação para o problema das $p$ medianas.

Uma vez obtido o conjunto de medianas $S$, faz-se necessário designar de maneira ótima (ou quase ótima) os pontos pertencentes ao conjunto $(V-S)$ a elas formando, desta maneira, os agrupamentos que serão atendidos pela frota disponível de veículos. A designação é realizada respeitando a capacidade da mediana (determinada pela capacidade de carregamento do veículo). Para a designação dos pontos, Gillet e Johnson (1976) propuseram um procedimento heurístico, conhecido como algoritmo de Gillet e Johnson.

A ideia básica do referido algoritmo é selecionar os pontos que apresentam a maior razão entre as distâncias às duas medianas mais próximas para serem designados com prioridade. Este procedimento prioriza a designação de pontos próximos às medianas.

Corrêa et al. (2004) propõem uma modificação no algoritmo original de Gillet e Johnson, que prioriza a designação dos pontos que possuem a maior diferença entre as distâncias das duas medianas mais próximas. Esta estratégia penaliza a designação de pontos à medianas distantes, caso a mediana mais próxima já tenha atingido sua capacidade. O algoritmo de Giller e Johson modificado é apresentado a seguir.

Parâmetros:

$t_{i}{ }^{l}$ : primeira mediana mais próxima ao ponto $i$

$t_{i}^{2}$ : segunda mediana mais próxima ao ponto $i$

$c_{i}^{l}$ : distância da primeira mediana mais próxima até o ponto $i$

$c_{i}^{2}$ : distância da segunda mediana mais próxima até o ponto $i$

Passo 1 Calcular a distância entre cada nó ainda não designado até cada um dos depósitos 
fictícios "medianas" correspondentes que ainda possuem capacidade;

Passo 2 Para cada nó $i$, obter $t_{i}{ }^{1}, t_{i}{ }^{2}, c_{i}{ }^{1}, c_{i}{ }^{2}$;

Passo 3 Para todos os nós $i$, calcular a diferença $d_{i}=c_{i}{ }^{1}-c_{i}{ }^{2}$;

Passo 4 Ordenar $i$ por $d_{i}$, em ordem decrescente;

Passo 5 Designar os pontos $i=1,2 \ldots$ até que $t_{i}{ }^{1}$ seja atingida;

Passo 6: Enquanto existir algum ponto $i$ não designado, volte ao passo 2; caso contrário, pare, a solução quase ótima (ou eventualmente a ótima) foi obtida.

\subsection{Procedimento para a construção de rotas}

Um dos possíveis procedimentos heurísticos para a construção de rotas para o $P C V$ é o algoritmo da inserção mais econômica. Este método constrói uma rota inicial e factível, atendendo a um conjunto de critérios que garantam certa eficiência para o refinamento da solução obtida. Uma descrição mais formal da heurística é apresentada por Steiner et al. (2000).

Passo 1. Comece com um sub-grafo consistindo somente do nó $p$;

Passo 2. Encontre o nó $m$ tal que distância $(p, m)$ seja mínima e forme a sub-rota $p-m-p$;

Passo 3. Encontre $i-j$ na sub-rota e $k$ que não esteja na rota, tal que:

distância $(i, k)+\operatorname{distância~}(k, j)$ - distância( $i, j)$ seja mínima e, então, insira $k$ entre $i$ e $j$;

Passo 4. Se todos os pontos do grupo já estiverem na rota, pare, a solução quase ótima (ou eventualmente a ótima) foi obtida. Caso contrário, volte ao passo 3.

\subsection{Algoritmos de melhorias de rotas}

Os algoritmos de melhorias de rotas buscam minimizar a distância total percorrida pelo caixeiro viajante pelo refinamento de uma solução inicial obtida a priori. A melhor classe conhecida de algoritmos é a heurística de trocas de arcos k-opt de Lin e Kernighan (1973), sendo as trocas 2-opt (2 arcos) e 3-opt (3 arcos) as mais utilizadas. Deve-se destacar que boas soluções iniciais são fundamentais para que se obtenham boas soluções finais (COSTA, 1997; CAMPOS, 2008).

Os métodos k-opt buscam a melhoria pela substituição de $k$ arcos no roteiro estabelecido anteriormente, isto é, $k$ arcos são removidos do roteiro e substituídos por outros $k$ arcos. Caso alguma melhoria seja detectada, a troca é aceita e o novo arco passa a compor a solução incumbente. Neste presente trabalho esta dinâmica se repete até que nenhuma troca resulte em melhoria.

Conforme apontado por Laporte (1999), o processo de melhoria k-opt termina em um mínimo local e possui ordem de complexidade $O(n k)$. Quanto maior for o valor de $k$, melhor será a 
solução, entretanto o esforço computacional requerido também será maior. Isto leva a um trade-off entre qualidade e tempo computacional. Dessa forma, trocas 4-opt e superiores ensejam um custo computacional muitas vezes superior à melhoria da solução obtida.

A Figura 1, a seguir, ilustra o caso particular da troca 2-opt, enquanto que a Figura 2, o caso da troca 3-opt. Para a troca de arcos 3-opt existirão sete diferentes possibilidades de novas rotas, mas três delas recaem em melhoria 2-opt (trocas 2, 4 e 7 da Figura 2), pois não houve substituição dos arcos 1-2, 3-4 e 4-6, respectivamente. Logo, na melhoria 3-opt, quatro novas alternativas de rotas são avaliadas a cada iteração.

Figura 1 - Melhoria 2-opt
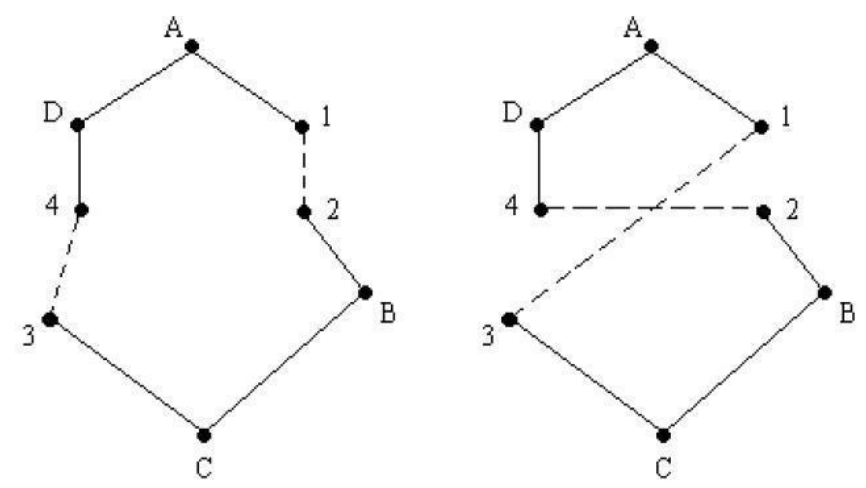

Fonte: Adaptado de Costa (1997)

Figura 2 - Melhorias 3-opt
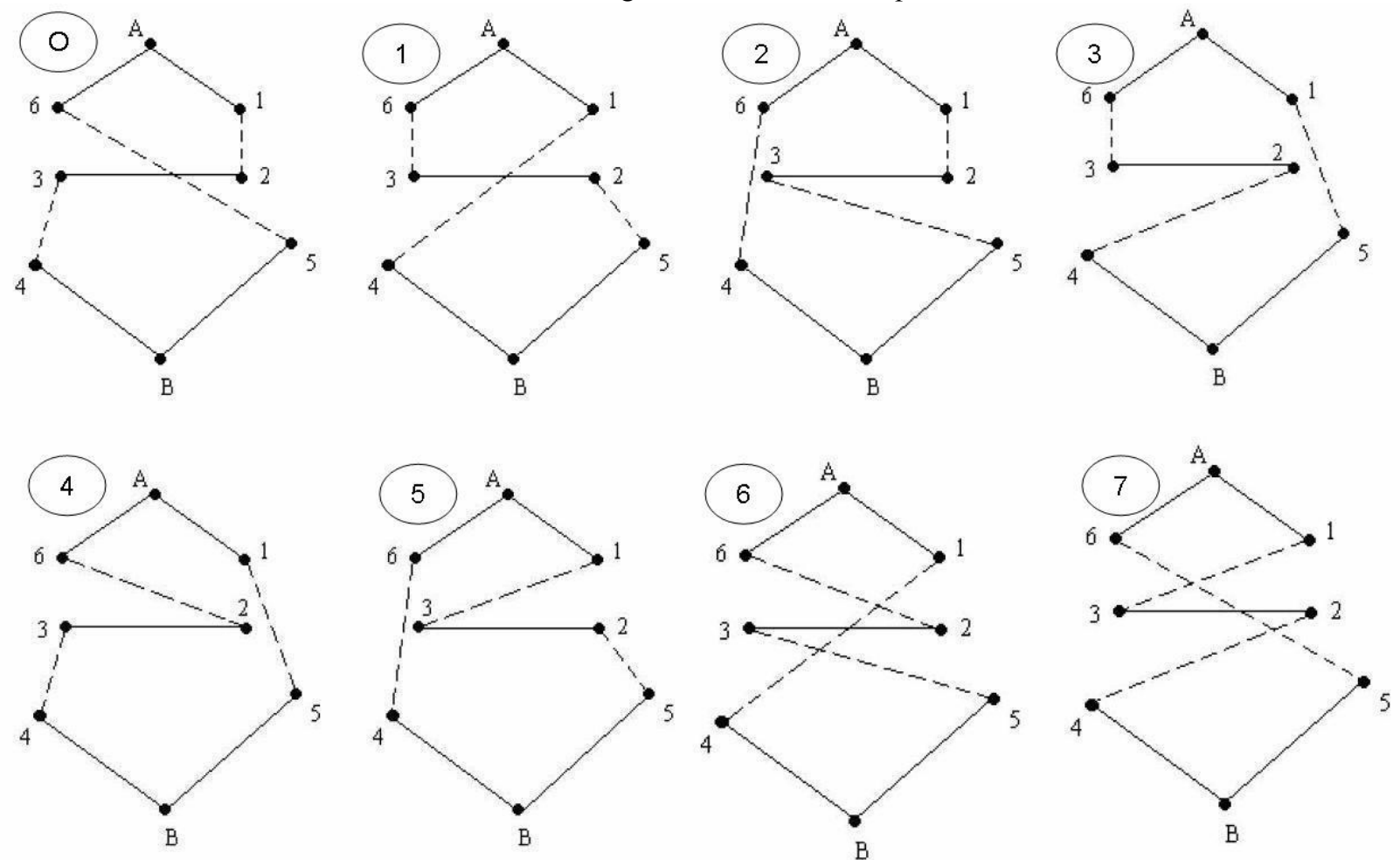

Fonte: Adaptado de Costa (1997)

\section{Abordagem proposta}


A estratégia para a resolução do PRVC inicia com o agrupamento dos clientes pelo algoritmo de Teitz e Bart (1968), ajustado a priori, para definição da quantidade de medianas conforme a quantidade de veículos. A designação dos pontos às medianas é feito pelo algoritmo de Gillet e Johnson (1976) modificado, formando os agrupamentos de clientes para serem roteirizados. Um novo ajuste, a posteriori a formação dos agrupamentos é aplicado. Neste trabalho considera-se a frota homogênea, porém o algoritmo proposto está generalizado para o caso de frota heterogênea. Ambos os ajustes configuram uma nova heurística, aqui denominada $k$-fit. $\mathrm{Na}$ seqüência cada agrupamento é roteirizado, sendo que a rota inicial é gerada pela heurística construtiva de inserção mais econômica e refinada pelas heurísticas de melhoria 2-opt e 3-opt. A abordagem completa é apresentada a seguir.

\section{Início do Método:}

Passo 1 - Definição da quantidade de medianas: Seja $n$ o número de clientes a serem atendidos, $d_{i}=\{i=1, \ldots, n\}$ as demandas de cada cliente e $C$ a capacidade de cada veículo;

1.1 - Dada a demanda total de todos os clientes $D$, soma-se a quantidade de veículos necessária para que se atenda a essa demanda. $\mathrm{O} k$-ésimo veículo que atende à demanda determina $k$ medianas.

Passo 2 - Agrupamento dos pontos pela heurística de Teitz e Bart (1968), com a quantidade $p$ de medianas obtidas no passo $1(p=k)$.

Passo 3 - Designação dos veículos para cada agrupamento: Aplica-se o algoritmo de Gillet e Johnson (1976) modificado.

Passo 4 - Ajuste dos agrupamentos:

4.1 - Aplica-se a heurística de inserção mais econômica na criação de um roteiro entre as medianas, iniciando e terminando a rota no depósito central. Criando uma ordem de pares agrupamento-veículo;

4.2 - Para cada $p$-agrupamento-veículo, decrescendo de $j=p$ até 2 , verifica-se se há excesso de demanda;

4.2.1 - Se sim, escolhe-se como pontos excedentes aqueles que estão mais próximos do agrupamento subseqüente (agrupamento $p=j$ - 1) e os insira neste agrupamento;

4.2.2 - Senão, avalie o próximo agrupamento $(p=j-1)$;

4.3 - Para cada $p$-agrupamento-veículo, crescendo de $j=1$ até $(p-1)$, verifica-se se há excesso de demanda;

4.3.1 - Se sim, escolhe-se como pontos excedentes aqueles que estão mais próximos do agrupamento subseqüente (agrupamento $p=j+1$ ) e os insira neste agrupamento;

4.3.2 - Senão, avalia-se o próximo agrupamento $(p=j+1)$. 
Passo 5 - Construção das rotas para cada agrupamento:

5.1 - Aplica-se a heurística construtiva de inserção mais econômica em cada agrupamento para obtenção de uma rota inicial;

5.2 - Aplica-se a heurística de melhoria 2-opt;

5.3 - Aplica-se a heurística de melhoria 3-opt.

\section{Fim do Método.}

Os passos 4.2 e 4.3 garantem que não haja excesso de demanda em cada veículo, viabilizando o carregamento. Da mesma forma, ao se definir a quantidade necessária de veículos para o atendimento do conjunto de rotas, também é garantido que a demanda é atendida totalmente. A heurística $k$-fit proposta é composta pelos passos 1 e 4 .

\section{Obtenção dos resultados}

O método proposto nesse trabalho foi testado sobre instâncias clássicas da literatura disponibilizadas nos sítio www.branchandcut.org. Tais instâncias foram utilizadas, dentre outros, nos trabalhos que empregam técnicas heurísticas e metaheurísticas propostas por Chen et al. (2006) e Campos e Mota (2000), além do estudo de Fukasawa et al. (2006), que apresenta um método exato para a resolução do problema. A razão da escolha dos métodos heurísticos e metaheurísticos reside no fato de seus testes terem sido realizados sobre o mesmo conjunto de instâncias avaliadas em Fukasawa et al. (2006). Assim, têm-se condições de comparar o desempenho tendo por base a solução ótima para a maioria das instâncias.

Os testes foram executados em um AMD Athlon TM 64 Dual Core, 2.10 Ghz com 896 MB de memória, sistema operacional Windows Vista. Embora o equipamento possua dois núcleos de processamento, foi utilizado apenas um deles. A linguagem de programação utilizada foi Visual Basic 6.0.

A Tabela 1, a seguir, apresenta os resultados obtidos para as instâncias testadas. Na primeira coluna tem-se a instância e nas duas colunas seguintes, a quantidade de pontos (clientes) e a quantidade de veículos, respectivamente. Nas colunas seguintes são apresentados os valores obtidos por Chen et al. (2006) (em relação à solução, coluna D, e tempo computacional, coluna T(s)) em suas propostas. O método AG 2-opt emprega algoritmos genéticos com melhoria 2-opt; DPSO-SA é um método metaheurísitco híbrido, que utiliza nuvem de partículas e recozimento simulado e, finalmente, SA que emprega isoladamente a metaheurística de recozimento simulado. A coluna seguinte apresenta os resultados para o método exato de Fukasawa (2006) e a coluna CM para a heurística de Campos e Mota (2000). Já a coluna BKS contém as melhores soluções, dentre os trabalhos apresentados. Por fim, as duas últimas colunas apresentam os resultados obtidos neste 
trabalho.

Tabela 1 - Resultados obtidos para as instâncias testadas

\begin{tabular}{|c|c|c|c|c|c|c|c|c|c|c|c|c|c|c|c|}
\hline \multirow{2}{*}{ Instância } & \multirow{2}{*}{$\mathbf{N}$} & \multirow{2}{*}{$\mathbf{K}$} & \multicolumn{2}{|c|}{ AG 2-opt ${ }^{1}$} & \multicolumn{2}{|c|}{ DPSO - $\mathbf{S A}^{1}$} & \multicolumn{2}{|c|}{$\mathbf{S A}^{1}$} & \multicolumn{2}{|c|}{ Fukasawa $^{2}$} & \multicolumn{2}{|c|}{$\mathrm{CM}^{3}$} & \multirow{2}{*}{ BKS } & \multicolumn{2}{|c|}{ PROPOSTO } \\
\hline & & & D & $\mathbf{T}(\mathbf{s})$ & D & $\mathbf{T}(\mathbf{s})$ & D & $\mathbf{T}(\mathbf{s})$ & D & $\mathbf{T}(\mathbf{s})$ & D & $\mathbf{T}(\mathbf{s})$ & & D & $\mathbf{T}(\mathbf{s})$ \\
\hline A-n33-k5 & 32 & 5 & 661 & 39,6 & 661 & 32,3 & 661 & 38,2 & - & - & - & - & 661 & 717,7 & 0,8 \\
\hline A-n46-k7 & 45 & 7 & 928 & 136,4 & 914 & 128,9 & 931 & 143,8 & 914 & 92 & - & - & 914 & 1087,6 & 2,8 \\
\hline A-n53-k7 & 52 & 7 & - & - & - & - & - & - & 1010 & 611 & - & - & 1010 & 1139,3 & 5,5 \\
\hline A-n54-k7 & 53 & 7 & - & - & - & - & - & - & 1167 & 1409 & - & - & 1167 & 1280,8 & 1,9 \\
\hline A-n60-k9 & 59 & 9 & 1360 & 295,5 & 1354 & 308,8 & 1363 & 286,3 & 1354 & 3080 & - & - & 1354 & 1508,5 & 7,8 \\
\hline A-n61-k9 & 60 & 9 & - & - & - & - & - & - & 1034 & 1883 & - & - & 1034 & 1182,5 & 2,5 \\
\hline A-n62-k8 & 61 & 8 & - & - & - & - & - & - & 1288 & 3102 & - & - & 1288 & 1447,5 & 4,7 \\
\hline A-n63-k10 & 62 & 10 & - & - & - & - & - & - & 1314 & 4988 & - & - & 1314 & 1454,3 & 12,3 \\
\hline A-n63-k9 & 62 & 9 & - & - & - & - & - & - & 1616 & 1046 & - & - & 1616 & 1762,5 & 2,9 \\
\hline A-n64-k9 & 63 & 9 & - & - & - & - & - & - & 1401 & 11254 & - & - & 1401 & 1579,77 & 2,5 \\
\hline A-n80-k10 & 79 & 10 & - & - & - & - & - & - & 1763 & 6464 & - & - & 1763 & 2015,1 & 6,9 \\
\hline B-n35-k5 & 34 & 5 & 955 & 46,9 & 955 & 37,6 & 960 & 58,4 & - & - & - & - & 955 & 974,19 & 1,2 \\
\hline B-n45-k5 & 44 & 5 & 762 & 129,3 & 751 & 134,2 & 760 & 123,5 & 751 & 16 & - & - & 751 & 771,0 & 2,9 \\
\hline B-n68-k9 & 67 & 9 & 1296 & 396,2 & 1272 & 344,3 & 1298 & 409,2 & 1272 & 87436 & - & - & 1272 & 1349,53 & 3,9 \\
\hline E-n51-k5 & 50 & 5 & 531 & 289,6 & 528 & 300,5 & 541 & 362,4 & - & - & 521 & 32,7 & 521 & 529,9 & 15,6 \\
\hline E-n76-k10 & 75 & 10 & - & - & - & - & - & - & - & - & 834 & 252,7 & 830 & 883,8 & 42,9 \\
\hline E-n76-k7 & 75 & 7 & 697 & 498,7 & 688 & 526,5 & 704 & 619,3 & - & - & 687 & 89,3 & 682 & 751,7 & 487,0 \\
\hline E-n76-k8 & 75 & 8 & - & - & - & - & - & - & - & - & 735 & 272,3 & 735 & 795,8 & 424,3 \\
\hline M-n101-k10 & 100 & 10 & 836 & 992,1 & 824 & 874,2 & 848 & 986,6 & - & - & 820 & 193,8 & 820 & 828,9 & 1447,9 \\
\hline M-n121-k7 & 120 & 7 & 1068 & 1643,1 & 1038 & 1733,5 & 1081 & 2729,5 & - & - & - & - & 1034 & 1101,5 & 441,1 \\
\hline P-n101-k4 & 100 & 4 & 706 & 1213,2 & 694 & 977,5 & 715 & 1964,9 & - & - & - & - & 681 & 746 & 133,3 \\
\hline P-n76-k4 & 75 & 4 & 605 & 528,4 & 602 & 496,3 & 612 & 489,6 & - & - & - & - & 593 & 655,8 & 227,5 \\
\hline
\end{tabular}

\footnotetext{
${ }^{1}$ Intel Pentium IV, CPU $1.80 \mathrm{GHz}, 256 \mathrm{MB}$ RAM

${ }^{2}$ Intel Pentium IV, CPU 2.4 GHz, 1 GB RAM

${ }^{3}$ Hardware não informado

Fonte: Os autores
}

Os campos assinalados com “_“ indicam que a instância não foi avaliada pelo trabalho indicado na respectiva coluna. Conforme se verifica na Tabela 1, o método proposto neste trabalho possui um tempo computacional consideravelmente reduzido para a maioria das instâncias testadas. Para as instâncias do grupo A e B, o tempo de processamento não ultrapassou 12,3 segundos. Instâncias acima de 70 pontos demandaram um tempo substancialmente maior pelo incremento dos movimentos 2-opt e 3-opt. Pela diferença de hardware existente entre este trabalho e as demais abordagens não é possível analisar os tempos comparativamente. Todavia os resultados demonstram que a heurística proposta produz bons resultados em relação aos trabalhos dos outros autores com tempos computacionais reduzidos. Registram-se ainda melhorias na solução das instâncias M-n101k10 e E-n51-k5 (AG-2-opt e SA em Chen et al. (2006)) . A Tabela 2, a seguir, apresenta os desvios para resultados obtidos em relação aos demais trabalhos. 
Tabela 2 - Análise comparativa das soluções e tempos de processamento

\begin{tabular}{ccccccccc}
\hline Instância & $\mathbf{N}$ & $\mathbf{K}$ & AG 2-opt & DPSO-SA & S.A & Fukasawa & CM & BKS \\
\cline { 4 - 7 } & & & GAP D & GAP D & GAP D & GAP D & GAP D & \\
\hline A-n33-k5 & 32 & 5 & $8,6 \%$ & $8,6 \%$ & $8,6 \%$ & - & - & $8,6 \%$ \\
A-n46-k7 & 45 & 7 & $17,2 \%$ & $19,0 \%$ & $16,8 \%$ & $19,0 \%$ & - & $19,0 \%$ \\
A-n53-k7 & 52 & 7 & - & - & - & $12,8 \%$ & - & $12,8 \%$ \\
A-n54-k7 & 53 & 7 & - & - & - & $9,8 \%$ & - & $9,8 \%$ \\
A-n60-k9 & 59 & 9 & $10,9 \%$ & $11,4 \%$ & $10,7 \%$ & $11,4 \%$ & - & $11,4 \%$ \\
A-n61-k9 & 60 & 9 & - & - & - & $14,4 \%$ & - & $14,4 \%$ \\
A-n62-k8 & 61 & 8 & - & - & - & $12,4 \%$ & - & $12,4 \%$ \\
A-n63-k10 & 62 & 10 & - & - & - & $10,7 \%$ & - & $10,7 \%$ \\
A-n63-k9 & 62 & 9 & - & - & - & $9,1 \%$ & - & $9,1 \%$ \\
A-n64-k9 & 63 & 9 & - & - & - & $12,8 \%$ & - & $12,8 \%$ \\
A-n80-k10 & 79 & 10 & - & - & - & $14,3 \%$ & - & $14,3 \%$ \\
B-n35-k5 & 34 & 5 & $2,0 \%$ & $2,0 \%$ & $1,5 \%$ & - & - & $2,0 \%$ \\
B-n45-k5 & 44 & 5 & $1,2 \%$ & $2,7 \%$ & $1,4 \%$ & $2,7 \%$ & - & $2,7 \%$ \\
B-n68-k9 & 67 & 9 & $4,1 \%$ & $6,1 \%$ & $4,0 \%$ & $6,1 \%$ & - & $6,1 \%$ \\
E-n51-k5 & 50 & 5 & $-0,2 \%$ & $0,4 \%$ & $-2,1 \%$ & - & $1,7 \%$ & $1,7 \%$ \\
E-n76-k10 & 75 & 10 & - & - & - & - & $6,0 \%$ & $6,5 \%$ \\
E-n76-k7 & 75 & 7 & $7,8 \%$ & $9,3 \%$ & $6,8 \%$ & - & $9,4 \%$ & $10,2 \%$ \\
E-n76-k8 & 75 & 8 & - & - & - & - & $8,3 \%$ & $8,3 \%$ \\
M-n101-k10 & 100 & 10 & $-0,8 \%$ & $0,6 \%$ & $-2,3 \%$ & - & $1,1 \%$ & $1,1 \%$ \\
M-n121-k7 & 120 & 7 & $3,1 \%$ & $6,1 \%$ & $1,9 \%$ & - & - & $6,5 \%$ \\
P-n101-k4 & 100 & 4 & $5,7 \%$ & $7,5 \%$ & $4,3 \%$ & - & - & $9,5 \%$ \\
P-n76-k4 & 75 & 4 & $8,4 \%$ & $8,9 \%$ & $7,2 \%$ & - & - & $10,6 \%$ \\
\hline Média & & & $5,7 \%$ & $6,9 \%$ & $4,9 \%$ & $11,3 \%$ & $5,3 \%$ & $9,1 \%$ \\
\hline
\end{tabular}

Fonte: Os autores

Em relação às abordagens metaheurísticas em Chen et al. (2009), observa-se que a heurística proposta neste trabalho apresentou um desvio médio de 5,7\% em relação ao algoritmo genético com melhoria 2-opt (com soluções melhores para as instâncias M-n101-k10 e E-n51-k5); 6,9\% para nuvens de partículas com recozimento simulado e 4,9\% em relação à metaheurística recozimento simulado pura. Já com relação à abordagem heurística de Campos e Mota (2000), o desvio médio foi de pouco mais de 5\%. Como esperado, os desvios foram maiores para abordagem exata de Fukasawa et al. (2006), com desvio médio superior à 11\% em relação à solução ótima. De forma global, o desempenho da heurística proposta apresentou soluções médias 9,1\% mais custosas em comparação com as melhores soluções conhecidas.

O fato das soluções obtidas pela heurística proposta neste estudo não se aproximarem muito dos melhores resultados da literatura (ótimos ou não), pode ser elucidado pelo emprego de técnicas simplificadas amplamente difundidas na literatura. Contudo, em situações reais de roteirização, o emprego dessas técnicas demonstrou-se eficiente visto que as soluções produzidas, embora não ótimas, podem ser consideradas de boa qualidade.

Deve-se destacar que o procedimento heurístico aqui proposto foi capaz de resolver todos os 
problemas, conforme resultados apresentados na Tabela 1 anterior, independentemente do número de pontos e em um tempo computacional bastante satisfatório.

\section{Conclusões}

Devido a sua alta aplicabilidade, o PRVC é um dos problemas mais estudados dentre os de otimização em redes. Embora a formulação exata para este problema seja simples, sua solução analítica é de alta complexidade, inviabilizando a resolução de situações para um grande número de pontos de demanda a serem visitados. Neste contexto, as heurísticas desempenham um papel importante como ferramenta de resolução.

No presente trabalho foi proposta uma estratégia em dois estágios para resolver este tipo problema. O primeiro deles agrupa os clientes conforme a demanda de cada um e, para isso desenvolveu-se uma heurística de ajuste, aqui denomina de $k$-fit, que foi aplicada em conjunto com o algoritmo clássico de Teitz e Bart (1968) conjugado com o algoritmo de Gillet Johson (1976). O segundo estágio trabalhou com a roteamento dos conjuntos formados pelo primeiro estágio e, para tanto, empregou-se a heurística de inserção mais econômica para a geração de rotas, além das heurísticas de melhorias $k$-opt para refinamento dessas soluções.

Para a validação do desempenho da heurística foram utilizadas instâncias avaliadas em outros trabalhos (exatos e heurísticos). Os resultados obtidos em relação ao tempo computacional foram satisfatórios, apresentando tempo de processamento bastante reduzido para a maioria das instâncias testadas. Houve também melhorias em relação à solução obtida para duas das instâncias em relação a um dos trabalhos comparados.

Destaca-se, em especial, é que o procedimento heurístico proposto foi capaz de resolver todos os problemas, independentemente do número de pontos. A heurística proposta mostra-se adequada para aplicações reais, pois é capaz de produzir boas soluções em tempo computacional reduzido.

\section{Abstract}

The Capacitated Vehicle Routing Problem is a well known approach in operations research to solve problems in distribution logistics. For its combinatorial complexity, the CVRP motivates the use of heuristic techniques for its resolution. In this sense, this paper presents a new heurist approach to solve the problem. The method employs a strategy in two stages. The first one builds clusters of customers according to the demand and the second stage builds routes for the costumers groups formed on the first stage. For the first stage, it was developed a heuristic evolving the classical clustering and assignment algorithms proposed by Teitz and Bart (1968) and Gillet and Johnson (1976), respectively. In the second stage, the initial routes were generated by the nearest insertion procedure and sequentially the routes were improved by the 2-opt and 3-opt heuristics. The proposed approach was tested on some classical instances from literature and the results were 
compared to some heuristics and exact procedures, producing interesting results, both in efficacy and efficiency.

Key-words: capacitated vehicle routing problem; heuristic procedures; demand point clustering.

\section{Referências}

ACHUTHAN, N.; CACCETTA, L.; HILL, S. An improved branch-and-cut algorithm for the capacitated vehicle routing problem. Transportation Science, v. 37, p. 153-169, 2003.

cross ref

BERGER, J.; BARKAOUI, M. A hybrid genetic algorithm for the capacitated vehicle routing problem. Lecture Notes in Computer Science, v. 2723, p. $646-656,2003$.

cross ${ }^{\text {ref }}$

CAMPOS, V.; MOTA, E. heuristic procedures for the capacitated vehicle routing problem. Computational Optimization and Applications, v. 16, p. $265-277,2000$.

cross ref

CAMPOS, D. S. Integração de problemas de carregamento e roteamento de veículos, com janela de tempo e frota heterogênea. 2008. 121 f. Tese (Doutorado em Engenharia de Produção) - Programa de Pós Graduação em Engenharia (Engenharia de Produção), Escola Politécnica da Universidade de São Paulo, São Paulo, 2008.

COSTA, D. M. B. Aplicação de algumas técnicas da pesquisa operacional na otimização de serviços postais. 1997. Dissertação (Mestrado em Métodos Numéricos em Engenharia), Universidade Federal do Paraná, Curitiba, 1997.

CORRÊA, E. et al. A genetic algorithm for solving a capacitated p-median problem, v.35, p. 373-388, 2004.

CHEN A.; YANG G.; WU Z. Hybrid discrete particle swarm optimization algorithm for capacitated vehicle routing problem. Journal of Zhejiang University Science, v. 7, p. 607-614, 2006.

cross

CHRISTOFIDES, N., Graph theory: an algorithmic approach. New York: Academic Press Inc, 1975.

CHRISTOFIDES, N.; MINGOZZI, A.; TOTH, P. Exact algorithms for the vehicle routing problem, based on spanning tree and shortest path relaxations. Mathematical Programming, v. 20, p. 255-282, 1981.

cross ${ }^{\text {ref }}$

DANTZIG, G.; RAMSER, R. The truck dispatching problem. Management Science, v. 6, p. 80-91, 1959.

cross ${ }^{\text {ref }}$

FISHER, M. L.; JAIKUMAR, R. A generalized assignment heuristic for vehicle routing. Networks, v.11, p.109-124, 1981.

cross ref

FUKASAWA, R. et al. Robust Branch-and-Cut-and-Price for the Capacitated Vehicle Routing Problem. Mathematical Programming, v. 106, n. 3, p. 491-511, 2006.

cross ref

GILLETT, B.; JOHNSON, J. Multi-terminal vehicle-dispatch algorithm. Omega, v. 4, p. 711-717, 1976.

cross ref

HAKIMI, S. L. Optimum Distribution of Switching Centers in a Communication Network and Some Related Graph Theoretic Problems. Operations Research, v. 13, p. 462-475, 1965.

cross ref

LAPORTE, G. et al. Classical and modern heuristics for the vehicle routing problem. International Transactions in Operational Research, v. 7, n. 4-5, p. 285-300, 1999.

cross ref 
LIN, S.; KERNIGHAN, B. W. An effective heuristic algorithm for the traveling salesman problem. Operations Research, v. 21, p. 498-516, 1973.

cross ${ }^{\text {ef }}$

LIN, S. W. et al. Applying hybrid meta-heuristics for capacitated vehicle routing problem. Expert Systems with Applications, v. 36, p. 1505-1512, 2009.

cross ref

PSINGER, D.; ROPKE, S. A general heuristic for vehicle routing problems. Computers \& Operations Research, v. 34, n. 8, p. 2403-2435, 2007.

cross ${ }^{\text {ref }}$

SARIKLIS D.; POWELL S. A heuristic method for the open vehicle routing problem. Journal of the Operational Research Society, v. 51, p.564-573, 2000.

cross ${ }^{\text {ref }}$

STEINER, M. T. A. et al. O problema de roteamento no transporte escolar. Pesquisa Operacional, v. 20, p. 83-99, 2000.

crossef

TEITZ, M. B.; BART, P. Heuristic methods for estimating the generalized vertex median of a weighted. Operations Research, v. 16, p. 955-961, 1968.

cross ref

ZHISHUO, L.; YUETING, C. Sweep based multiple ant colonies algorithm for capacitated vehicle routing problem. In: IEEE INTERNATIONAL CONFERENCE ON E-BUSINESS ENGINEERING (ICEBE'05), Beijing, 2005. IEEE Computer Society, Technical Committee on Electronic Commerce (TCEC). Proceedings... v. 387 - $394,2005$.

\section{Dados dos autores:}

Nome completo: Thiago André Guimarães

Filiação Institucional: Universidade Federal do Paraná - UFPR

Departamento: Programa de Pós Graduação em Métodos Numéricos em Engenharia - PPGMNE

Função ou cargo ocupado: Mestrando

Endereço completo para correspondência:

Rua Mateus Leme, 1244, apto. 201,

Centro Cívico, Curitiba, Paraná, Brasil, CEP: 80.530-010.

Telefones: +55 (41) 96722843; +55 (41) 30222843

\section{Nome completo: Cassius Tadeu Scarpin}

Filiação Institucional: Universidade Federal do Paraná - UFPR

Departamento: Programa de Pós Graduação em Métodos Numéricos em Engenharia - PPGMNE 
Função ou cargo ocupado: Doutorando

Endereço completo para correspondência: Rua Professor João Soares Barcelos, 80 A, Hauer, Curitiba, Paraná, CEP: 81630-060

Telefones: +55 (41) 91072942; +55 (41) 30397239

Nome completo: Maria Teresinha Arns Steiner

Filiação Institucional: Universidade Federal do Paraná - UFPR

Departamento: Programa de Pós Graduação em Métodos Numéricos em Engenharia - PPGMNE e Programa de Pós-Graduação em Engenharia de Produção - PPGEP

Função ou cargo ocupado: Professora Sênior (Associado III); Pesquisadora nível 1C do CNPq

Endereço completo para correspondência: Rua Padre Anchieta, 1231, apto. 31, 80.730-000 Curitiba, PR

Telefones: +55 (41) 99946359; +55 (41) 33613218

Enviado em: 22/11/2010

Aprovado em: 20/09/2011 